\title{
A new stage of the Evolutionary and Institutional Economics Review
}

\author{
Kiichiro Yagi ${ }^{1}$ Yuji Aruka ${ }^{2}$ Takahiro Fujimoto ${ }^{3}$
}

Published online: 5 June 2015

(C) Japan Association for Evolutionary Economics 2015

At the end of the nineteenth century, in 1898, Thorstein Veblen asked why economics is not an evolutionary science. In the same year, Alfred Marshall wrote that the mecca of economists is economic biology. Thus, the academic interest in the direction of evolutionary economics is not new. The evolutionary or biological direction, then, meant the dynamics of human nature in its interaction with institutional environment. It suggested the need for synthesis of historical and theoretical analysis that social sciences in the preceding century had contributed.

This hope of evolutionary synthesis was not fulfilled in the twentieth century. A counterdirection of neo-classical economics that sought a rational construction of pure economics based on methodological individualism dominated academia. All of the social elements were expelled from the economic theory together with the empirical reality. Economics in the twentieth century was a lonely queen who isolated herself from other social sciences to say nothing of natural sciences. It was only in the late decades of the century that the direction of evolutionary synthesis regained its momentum.

However, this retarded resumption had its own advantage, as it could assimilate various recent scientific advances. Not only the progress in molecular biology but

Kiichiro Yagi

yagi@econ.setsunan.ac.jp

Yuji Aruka

aruka@tamacc.chuo-u.ac.jp

Takahiro Fujimoto

fujimoto@e.u-tokyo.ac.jp

1 Faculty of Economics, Setsunan University, Osaka, Japan

2 Faculty of Commerce, Chuo University, Tokyo, Japan

3 Faculty of Economics, The University of Tokyo, Tokyo, Japan 
also in the science of complexity opened a new horizon for evolutionary thinking. At present many evolutionary economists utilize nonlinear dynamics, computer simulation, and gaming experiments in their research. On the other hand, most traditional research areas of empirical or historical direction wait for the new interpretation from the synthetic view of evolutionary economics.

In March 1997, nearly a century after Veblen and Marshall's appeal for evolutionary economics, over a hundred economists gathered in Kyoto to join this innovative movement by founding the Japan Association for Evolutionary Economics (JAFEE). Soon this association grew into one of the most active forums of heterodox economists in Japan that has at present about 400 members. It was this Japan-based association that launched this journal, Evolutionary and Institutional Economics Review (EIER), in 2004. From the beginning EIER was an open and refereed journal with an international editorial board. In spite of all the limitations from financial means to insufficient experience in international publication, EIER published in its first 11 volumes some 150 articles and notes. These are now available from the archive of the back numbers on EIER's Website (http://www.springer.com/economics/economic+theory/journal/40844). It also acquired a non-negligible position in worldwide heterodox economics.

Now, from its 12 th volume, EIER is being published by Springer Japan, keeping the linkage with JAFEE intact. This move is a great opportunity for EIER, since it can now utilize the advanced technology and qualified skills of one of the most renowned academic publishers. Ranked among the Springer series of journals, EIER must maintain the rigor and vigor to participate in the advances of social sciences. In the area of institutional and evolutionary economics remain many unexplored frontiers. Thus, EIER asks for the collaboration of all the ambitious researchers in economics and related sciences to advance the evolutionary synthesis in economics.

May 2015

Evolutionary and Institutional Economics Review

Kiichiro Yagi, Editor-in-Chief

Yuji Aruka and Takahiro Fujimoto, Coordinating Editors 